\title{
RESEARCH
}

Open Access

\section{Eat, play, live: a randomized controlled trial within a natural experiment examining the role of nutrition policy and capacity building in improving food environments in recreation and sport facilities}

Dana Lee Olstad ${ }^{1}$, Kim D. Raine ${ }^{2}$, Rachel J. L. Prowse ${ }^{2}$, Dona Tomlin³ ${ }^{3}$ Sara F. Kirk ${ }^{4}$, Jessie-Lee D. Mclsaac ${ }^{5}$, Louise C. Mâsse ${ }^{6}$, M. Susan Caswell ${ }^{7}$, Rhona M. Hanning ${ }^{7}$, Todd Milford ${ }^{8}$ and Patti-Jean Naylor ${ }^{3^{*}}$ (D)

\begin{abstract}
Background: Recreation and sport facilities often have unhealthy food environments that may promote unhealthy dietary patterns among children. In response, some Canadian provinces have released voluntary nutrition guidelines for recreation and sport facilities, however implementation has been limited. Organizational capacity building may overcome barriers to implementing guidelines. Eat, Play, Live was a randomized controlled trial embedded within a natural experiment that tested the impact of an 18 month capacity building intervention (CBI) in enhancing implementation of provincial nutrition guidelines, and whether nutrition guidelines were associated with positive changes. Primary outcomes were facility capacity, policy development and food environment quality.

Methods: Recreation and sport facilities in three guideline provinces were randomized into a guideline + CBI (GL + $\mathrm{CBI} ; n=17)$ or a guideline only comparison condition (GL-ONLY; $n=15)$. Facilities in a province without guidelines constituted a second comparison condition (NO-GL; $n=17$ ). Facility capacity, policy development, and food environment quality (vending and concession) were measured and compared at baseline and follow-up across conditions using repeated measures ANOVA and Chi-square statistics. Healthfulness of vending and concession items was rated as Do Not Sell (least nutritious), Sell Sometimes or Sell Most (most nutritious).

Results: There were significant time by condition effects, with significant increases in facility capacity (mean \pm SD: $30.8 \pm 15.6 \%$ to $62.3 \pm 22.0 \% ; p<0.01$ ), nutrition policy development (17.6\% developed new policies; $p=0.049$ ), overall quality of the concession food environment (14.7 \pm 8.4 to $17.5 \pm 7.2 ; p<0.001)$, and in the proportion of Sell Most (3.7 $\pm 4.4 \%$ to $11.0 \pm 9.0 \% ; p=0.002)$ and Sell Sometimes vending snacks ( $22.4 \pm 14.4 \%$ to $43.8 \pm 15.8 \%$; $p<0.001)$ in $G L+C B I$ facilities, with a significant decline in Do Not Sell vending snacks $(74.0 \pm 16.6 \%$ to $45.2 \pm$ 20.1\%; $p<0.001)$.

\footnotetext{
*Correspondence: pjnaylor@uvic.ca

${ }^{3}$ School of Exercise Science, Physical and Health Education, University of

Victoria, PO Box 3015 Stn CSC, Victoria, BC V8W 3P1, Canada

Full list of author information is available at the end of the article
}

(c) The Author(s). 2019 Open Access This article is distributed under the terms of the Creative Commons Attribution 4.0 International License (http://creativecommons.org/licenses/by/4.0/), which permits unrestricted use, distribution, and reproduction in any medium, provided you give appropriate credit to the original author(s) and the source, provide a link to the Creative Commons license, and indicate if changes were made. The Creative Commons Public Domain Dedication waiver (http://creativecommons.org/publicdomain/zero/1.0/) applies to the data made available in this article, unless otherwise stated. 


\begin{abstract}
(Continued from previous page)
Conclusions: Significant improvements in facility capacity, policy development and food environment quality occurred in recreation and sport facilities that were exposed to nutrition guidelines and participated in a CBI. Outcomes did not improve in facilities that were only passively or not at all exposed to guidelines. Ongoing capacity building may enhance implementation of voluntary nutrition guidelines, however food environments remained overwhelmingly unhealthy, suggesting additional scope to enhance implementation.
\end{abstract}

Trials registration: Clinical trials registration (retrospectively registered): ISRCTN14669997 Jul 3, 2018.

Keywords: Children, Food environments, Recreation and sport facilities, Policy, Capacity building, Public health

\section{Introduction}

Sports and unhealthy food and drink - the former promotes health, while the latter can undermine it; yet in many cases the two are closely associated. This is no less the case in Canada, where publicly funded recreation and sport facilities (community facilities with physical activity-related infrastructure and programming) often have unhealthy food environments, despite their mandate to promote health [1-4]. The preponderance of unhealthy food environments in these facilities is a concern because a majority of users are children who, by virtue of their immaturity, may be particularly vulnerable to negative environmental exposures [5, 6]. Indeed, whereas in adults positive health behaviours tend to cluster $[7,8]$, children involved in sport actually consume more sugar sweetened beverages, fast food and energy relative to children who are less active [9]. Moreover, compared to their mothers, children are more likely to consume unhealthy foods during periods of physical activity [10]. These differences might plausibly relate to the unhealthy nature of the food environment in many community recreation and sports settings and suggest a need for remedial action.

The socioecological model can provide a foundation for understanding potential leverage points to improve children's dietary intakes [11]. Policy constitutes the outermost sphere of the socioecological model because it establishes the default conditions for all other levels; it shapes organizational and community environments which in turn influence individuals' personal capabilities and resources. Given its pervasive influence, public policy governing food availability, accessibility and promotion has therefore emerged as a top priority to improve children's food environments worldwide [12]. However, while action to develop and implement nutrition policies has occurred in schools, other public settings such as recreation and sports facilities have received limited attention. Notably, three Canadian provinces (equivalent to states) stand out as among few jurisdictions worldwide that have taken action by releasing nutrition guidelines, albeit of a voluntary nature, for recreation and sport facilities [13-15]. Although voluntary guidelines may lack the potency of mandatory policies, we have previously shown that recreation and sport facilities located in provinces with nutrition guidelines had healthier food environments than facilities in a province without nutrition guidelines (unpublished observations). Nevertheless, food environments remained overwhelmingly unhealthy in all cases, suggesting that voluntary nutrition guidelines alone are not sufficient to create truly healthy food environments, and that additional supports are required to overcome barriers to their implementation $[2,4,16,17]$.

Organizational capacity building within recreation and sport facilities offers potential to overcome barriers to implementing provincial nutrition guidelines (e.g. perceived lower profitability of healthier items, limited knowledge and skills to implement nutrition guidelines, minimal stakeholder buy-in and cooperation $[1,2,4,18$, 19]). Previous capacity building interventions (CBI) have supported organizations in successfully addressing health-related issues by enhancing local commitment, knowledge, skills, leadership, structures and systems [20]. Because they rely largely on existing resources and supports, CBIs do not require new, costly infrastructure or bureaucracies, thereby enhancing their feasibility. We have previously shown that when provincial nutrition guidelines were in place, 8 months of capacity building activities focussed on improving problem recognition, engaging key stakeholders, and providing training, resources, and supports were associated with positive change in recreation and sport facility capacity, policy development and food environments [2]. Given promising results from this quasi-experimental controlled study (i.e. facilities self-selected to participate in a CBI and were compared to facilities that volunteered to act as comparison facilities), we perceived a need to study the impact of a more comprehensive, longer CBI scaled up to a national level, and to use a stronger randomized, controlled design capable of supporting causal inference.

Thus, the purpose of the Eat, Play, Live (EPL) trial was to provide longitudinal evidence of the impact of an 18 month CBI in enhancing implementation of provincial nutrition guidelines across multiple provincial contexts. 
Primary outcomes were recreation and sport facility capacity, policy development and food environments. Figure 1 presents the hypothesized pathway from intervention to outcomes. We also examined whether nutrition guidelines were associated with positive change in these outcomes over time. Our overall aim was to test whether a relatively small investment in building capacity could protect government's much larger investment in developing and disseminating voluntary nutrition guidelines, and the level of implementation that could realistically be achieved within a voluntary policy framework.

\section{Methods}

\section{Study design and context}

EPL was a RCT embedded within a natural experiment that leveraged differences in nutrition policy/guidelines for recreation and sport facilities among Canadian provinces. Policies are defined as a "relatively stable, purposive course of action followed by an actor or set of actors in dealing with a problem or matter of concern" [21] and can include both formal and informal rules, laws and regulations [22]. The terms policy and guidelines are therefore used interchangeably throughout this paper. Participating facilities in the three Canadian provinces with nutrition guidelines for recreation and sport facilities at the time of the study (British Columbia $=\mathrm{BC}$, Alberta $=\mathrm{AB}$, Nova Scotia $=$ NS) [13-15] were randomized to an 18 month CBI or a guideline only comparison condition (i.e. no $\mathrm{CBI}$ ). Facilities in a province that did not have nutrition guidelines for recreation and sport facilities $($ Ontario $=$ $\mathrm{ON}$ ) constituted a second comparison condition that was not randomized (Fig. 2). ON was selected as a no guideline comparison province as it provided the closest match in terms of population size, urban/rural mix, economic activity, language and culture.

The study received ethical approval from the Health Research Ethics Boards at the University of Victoria, the University of British Columbia, the University of Alberta, the University of Waterloo and Dalhousie University. Managers of recreation facilities agreed to their facilities' participation. This manuscript adheres to CONSORT (Additional file 1) and TIDieR (Additional file 2) reporting standards.

\section{Recruitment}

Recreation and sport facilities were recruited by linking with each province's respective Recreation and Parks Association (or a similar organization) to initiate website postings, email their members and provide presentations at their annual meetings. Publicly funded municipal recreation and sport facilities were eligible to participate if they: 1) Had not been involved in an intervention to improve their food environment since 2010; 2) Offered food/beverages through vending machines and/or a concession (analogous to cafeterias, canteens and kiosks); 3) Had the ability to change their food environment; and 4) Offered recreational programming, preferably to children. Facilities located in close proximity to the coordinating Universities were preferred. A total of 286 facilities indicated an interest in participating, of which 145 returned our phone calls/emails. Of the 145 facilities contacted, 75 facilities were eligible and 49 facilities enrolled (65\%) (Fig. 3). Reasons for nonparticipation were: 11 lacked staff capacity, two were not interested in participating in research, one was unwilling to potentially be allocated to a comparison condition, one was afraid of losing revenue and 11 provided no reason.

\section{Allocation to condition}

Three parallel groups were included (Fig. 2). Following collection of baseline data, an independent researcher used a 1:1 allocation ratio to randomize the 32 facilities located in guideline provinces into a guideline $+\mathrm{CBI}$ $(n=17)$ or a guideline only comparison condition $(n=$ 15). A computer-generated randomization sequence stratified by province, food service types (i.e. vending only, concession only, mixed vending and concession), and participation in pre-2010 interventions was used. One extra facility was allocated to the guideline + CBI group in $\mathrm{AB}$ and NS, as there was an uneven number of facilities in these provinces. Allocation concealment was ensured via secure storage of the randomization sequence separately from the facility database, and was only accessible to the researcher who performed the randomization. All 17 facilities in the province of $\mathrm{ON}$ constituted the no guideline comparison group. The unit of randomization and analysis was individual recreation and sport facilities. Facilities could not be blinded to group assignment.

1) The no guideline comparison group (NO-GL) consisted of facilities in $\mathrm{ON}$, a province that did not have nutrition guidelines for recreation and sport facilities. These facilities had the potential to be passively exposed to all publicly available nutrition guidelines and resources (e.g. online toolkits), however a manipulation check confirmed that prior to the study none were aware of guidelines in other provinces, nor did they investigate them during the course of the study. These facilities were not randomized but were assigned 'by nature' to condition, and thus did not self-select their condition.

2) The guideline only comparison group (GLONLY) consisted of facilities in $\mathrm{BC}, \mathrm{AB}$ and NS that were passively exposed to their respective provincial nutrition guidelines and associated 
resources, along with all other publicly available guidelines and resources. These facilities were randomized to condition.

3) The guideline + capacity building intervention group $(\mathrm{GL}+\mathrm{CBI})$ consisted of facilities in $\mathrm{BC}, \mathrm{AB}$ and NS. These facilities were subject to the same provincial context and guideline exposures as the guideline only group, and received an actively delivered 18 month CBI. These facilities were randomized to condition.

\section{Intervention}

\section{Provincial nutrition guidelines}

Voluntary nutrition guidelines for recreation and sport facilities were introduced at different times in the three Canadian provinces, with NS in early [15], AB at mid [14] and $\mathrm{BC}[13]$ at a later stage of guideline implementation at the time of the study. All three provincial guidelines provide a basis to classify the healthfulness of foods using nutrient profiling schemes, along with guidance as to how facilities can support healthy eating by increasing the availability, accessibility and promotion of healthier foods. There is moderate to good agreement between nutrition profiling schemes in $\mathrm{BC}, \mathrm{AB}$ and NS [23], although some aspects of some schemes were recently modified by their respective governments. Facilities in each province could access a variety of province-specific supports to facilitate guideline implementation, which primarily consisted of online toolkits and implementation guides.

\section{Capacity building intervention model, process and components}

The overall CBI model, previously developed and refined in $B C[2,24]$, was modeled after the linking system approach $[25,26]$. The CBI was designed to link researchers (the 'resource group') with recreation facility staff (the 'user group'). A Provincial Coordinator in each guideline province served as the 'linking agent/knowledge broker' and provided training and technical support as facilities created localized facility action plans. Provincial Coordinators included two Registered Dietitians/PhD Candidates and one MSc-level Research
Associate. All three were trained in health promotion and nutrition and had conducted previous research in recreation and sport facilities. Provincial Coordinators also linked facilities with resources that were available to support their work (e.g. online toolkits, published evaluations, public health dietitians), and provided feedback on their progress. In this way, the Provincial Coordinator facilitated the translation of knowledge into action [27]. It was envisioned that lessons learned by the 'user group' would influence further actions within and across sites because of opportunities for cross-site sharing embedded within the CBI.

The specific elements of the CBI were informed by the implementation science literature $[25,26,28]$ and our previous research in recreation and sport facilities $[1,2,4,24$, 29-31] (Table 1). It represents the combination of elements that proved to be both acceptable to stakeholders and crucial to intervention effectiveness. The purpose of the CBI was to build local stakeholder capacity for implementing provincial nutrition guidelines through a series of five suggested steps executed over the course of 18 months: 1) Receive a half-day of province-specific training from Provincial Coordinators (facility managers or other designates were trained in an in-person group setting immediately after randomization); 2) Form a local planning team; 3) Review baseline evaluation report (i.e. facility capacity and food environment quality); 4) Create an individualized facility action plan; and 5) Implement, monitor and adjust the action plan. The implementation process (i.e. steps $2-5$ ) was to unfold organically within individual facilities with limited involvement from researchers. Researchers did not prescribe certain changes; instead, facilities created, revised and executed their own contextualized action plans consistent with their priorities. Provincial Coordinators provided on-demand support and maintained regular contact with facilities (email, phone, in-person) at least monthly. Seed funds in the amount of $\$ 1000 \mathrm{CAD}$ were also provided 3 months after training to each facility to invest in their food change activities, and all study-associated travel was reimbursed. Provincial (2, 6, 12 months) teleconferences supported cross-site sharing. At the end of the study, NO-GL and GLONLY facilities were offered a CBI consisting of the initial

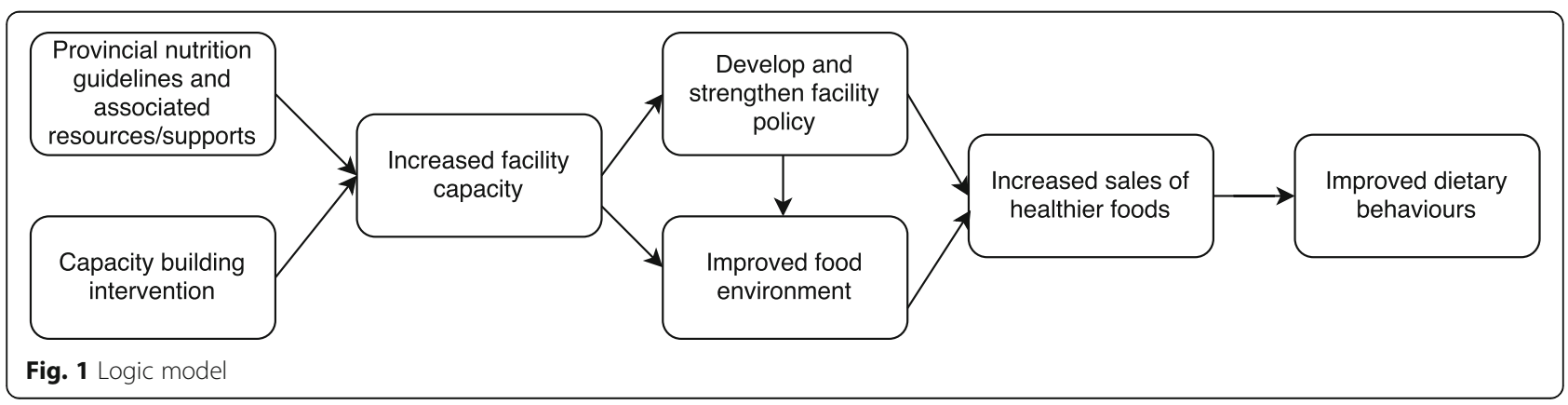




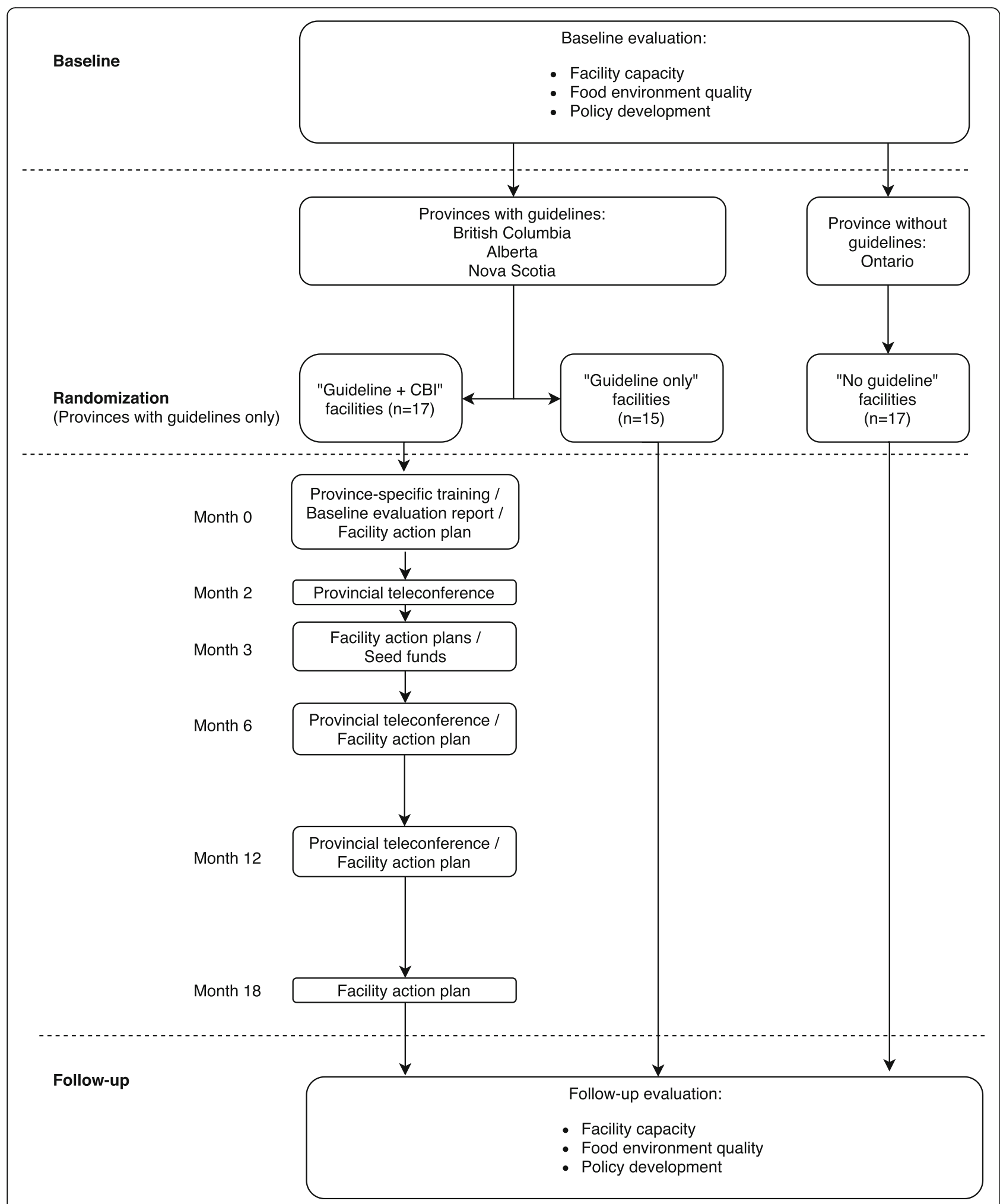

Fig. 2 Study flow chart 


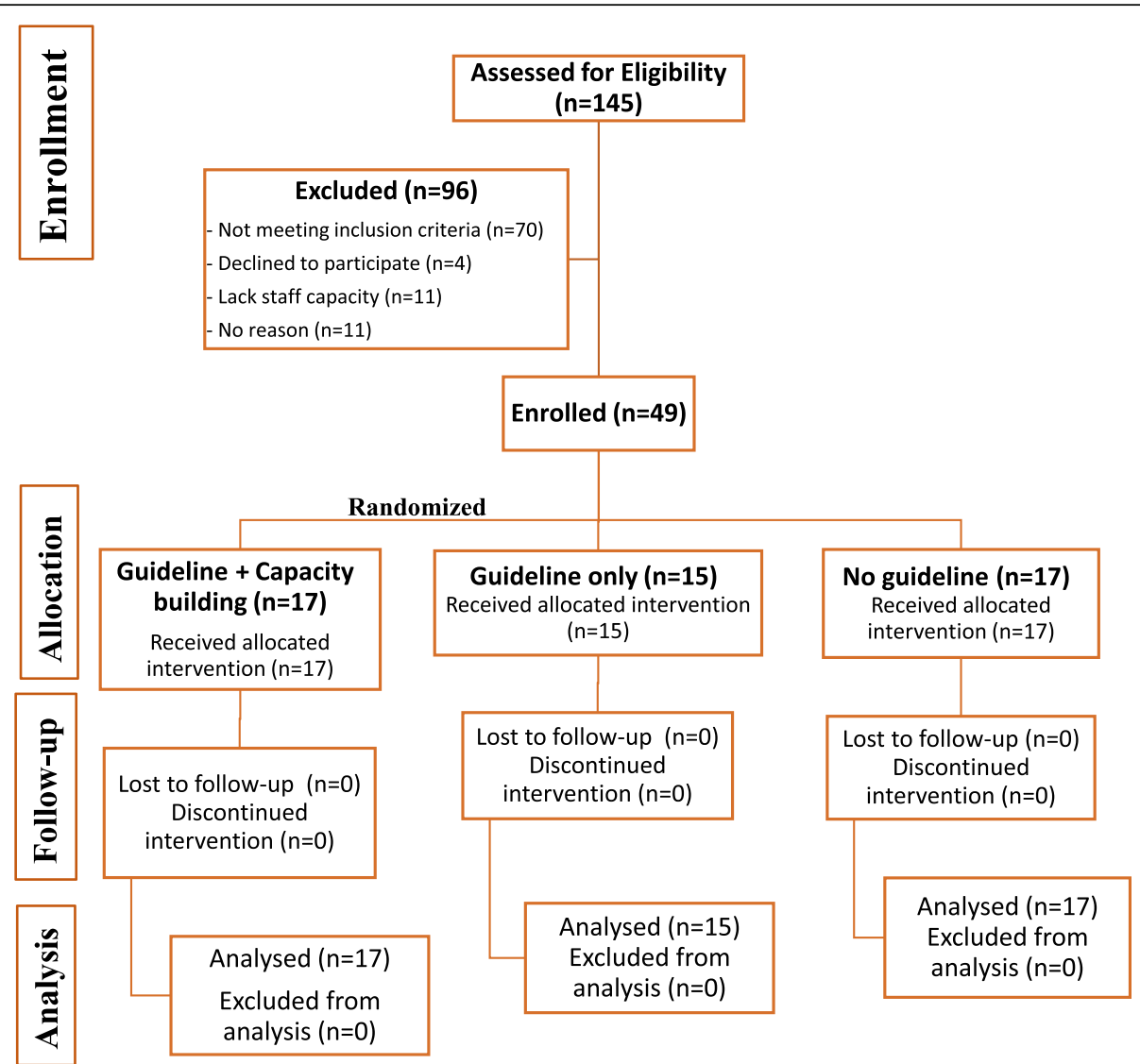

Fig. 3 CONSORT flow diagram

CBI training session, access to an online toolkit and 6 months of on-demand support. Many of the CBI resources have now been made publicly available at: https://stayactiveeathealthy.ca/website-resources/.

\section{Sample size calculation}

Forty-two facilities in total were required to detect a medium to large effect $(0.5)$ in vending product health profiles with $80 \%$ power and $\alpha=0.05$ ( $G$ * Power v 3.1).

\section{Data collection}

Primary study outcomes reported in this paper were assessed at baseline (December, 2015 - May, 2016) and 18 months later in all facilities, including: 1) Facility capacity; 2) Policy development; and 3) Food environment quality.

\section{Facility capacity}

Facility capacity to support the provision and promotion of healthy foods was assessed using a modified Facilities Assessment questionnaire that we have successfully used to detect differences in facility capacity in previous investigations [2, 24] (Additional file 3). Given that the current study included objective measures of the food environment, questions related to the perceived quality of the food environment were omitted. A representative from each facility answered 10 questions (response options: $0=$ not in place; $1=$ under development; $2=$ partially in place/could be improved; $3=$ fully in place) regarding their facility's status with respect to strategic planning (e.g. policies and/or plans are in place) and communication and education (e.g. staff/volunteers receive nutrition training) for a total possible score of 30 .

\section{Nutrition policy development}

Policy development at the facility level was assessed using one item from the Facilities Assessment questionnaire that asked whether a written nutrition policy/ guideline/plan was: not in place, under development, partially in place, or fully in place (Additional file 3). Responses were dichotomized as: fully/partially in place vs under development/not in place.

\section{Food environment - vending machines}

In each facility, a Research Assistant used a reliable (testretest and inter-rater reliability $\geq 0.88$ ) four-step process to audit each of two randomly selected snack and beverage 
vending machines [24] (Additional file 4). Information on product brand, variety/type, size and flavour was recorded for each item to calculate the proportion of products classified as: Sell Most (i.e. nutrient-rich; lower in sodium, sugar and fat), Sell Sometimes (i.e. source of essential nutrients; higher in sodium, sugar, and/or fat), and Do Not Sell (i.e. energy-dense and nutrient-poor; high in sodium, sugar, and/or fat) [13], using an online automated classification tool [32]. Products were classified using BC's food classification scheme to provide a consistent basis for cross-province comparisons.

\section{Food environment - concessions}

The majority of facilities had a single concession. When more than one concession was present, the one able to provide itemized sales data was audited (these data are forthcoming); otherwise a target concession was randomly selected. For each target concession, we calculated the proportion of packaged Sell Most, Sell Sometimes and Do Not Sell products as previously described, and subsequently assessed overall food environment quality using an adapted version of the Nutrition Environment Measures Survey-Restaurant reduced item audit (rNEMS-R; fast casual and fast food versions; Additional file 5) [33]. Scores on the rNEMS-R do not differ significantly from the full valid and reliable NEMS-R [34]. Higher scores (possible range for fast casual: -4.02 to +48.27 ; fast food: -10.17 to +51.35$)$ indicate greater availability, supports for (e.g. signage and promotions), and lower cost of healthy options [33]. We also assessed the availability of specific 'marker' foods (e.g. fruit, vegetables, regular and baked potato chips, French Fries) and food preparation equipment (e.g. deep fat fryer, grill) listed in the full NEMS-R [34], along with alcohol, as indicators of the healthfulness of food environments.

\section{Data analysis}

Descriptive characteristics were calculated as proportions, means and ranges. Repeated measures ANOVA assessed differences in facility capacity and food environments across the three conditions over time, adjusting for baseline values. Values for vending machines of the same type were averaged within facilities. The effect of time by condition was the main outcome of interest. Where there were significant main effects of time by condition, pairwise comparisons using Bonferroni correction were conducted. Associations between condition and policy development (fully/partially in place vs under development/not in place) and availability of marker foods and food preparation equipment (healthy change/maintained healthy status vs not) over time were examined using Chisquare Tests of Association.
For all comparisons, differences in outcomes between GL $+\mathrm{CBI}$ and GL-ONLY facilities captured the effect of the CBI, whereas comparisons of these groups with NOGL facilities captured changes associated with provincial nutrition guidelines, with or without capacity building. All analyses were by originally assigned groups. The proportion of missing data was very low and thus a complete case analysis was conducted under a missing completely at random (MCAR) assumption, which was a reasonable assumption given reasons for missingness described below. Findings were unchanged when the five missing facility capacity scores were carried forward from baseline to follow-up. SPSS (version 24; IBM Corporation, Armonk, NY) was used for all analyses, with $p<0.05$ indicating significant differences.

\section{Sensitivity analyses}

As part of the CBI, GL + CBI facilities created their own facility-specific change plans. As a result, some facilities chose to focus their efforts on improving the nutritional quality of their vending machine items, whereas others focussed on improving the nutritional quality of their concession items, rather than attempting to change both simultaneously. Sensitivity analyses therefore examined whether findings differed when only those facilities that were actively working to improve the proportion of healthy vending $(n=11)$ or concession $(n=7)$ items, respectively, were included in the analyses.

\section{Results}

\section{Descriptive characteristics}

All 49 facilities completed the study and provided follow-up data (note that not all facilities had snack and beverage vending machines and a concession, and some concessions did not sell packaged beverages and others did not sell packaged snacks). Five facilities did not report facility capacity scores at follow-up (citing lack of time). Two concessions closed during the course of the study. Moreover, two concessions stopped selling packaged snacks and one stopped selling packaged beverages between baseline and follow-up.

Table 2 presents baseline descriptive characteristics for facilities. At baseline, NO-GL facilities had lower capacity (Table 3 ) and poorer quality food environments (Tables 4 and 5) relative to facilities in guideline provinces.

\section{Facility capacity}

There was a significant effect of time $(\mathrm{F}=28.618$; $p<0.001)$ and time by condition $(\mathrm{F}=7.908 ; p=0.001)$ for facility capacity scores (Table 3 ). Overall facility capacity (\% ideal score) increased from $30.8 \pm 15.6 \%$ to $62.3 \pm$ $22.0 \%$ in GL + CBI facilities compared to GL-ONLY 
$(23.8 \pm 21.7 \%$ to $24.9 \pm 26.1 \% ; p<0.01)$ and NO-GL facilities $(15.5 \pm 12.9 \%$ to $33.3 \pm 14.3 \%$; $<<0.01)$.

\section{Nutrition policy development}

Between baseline and follow-up, 17.6\% of GL + CBI facilities developed new written facility nutrition policies whereas no GL-ONLY or NO-GL facilities developed new written policies $\left(X^{2}=6.015, p=0.049\right)$.

\section{Food environment - vending machines}

There was a significant effect of time for Do Not Sell vending beverages $(\mathrm{F}=4.951, p=0.03)$. There were no significant time by condition effects for vending beverages (Table 4).

There was a significant effect of time for Do Not Sell $(\mathrm{F}=13.687 ; \mathrm{p}=0.001)$ and Sell Sometimes $(\mathrm{F}=15.995$; $\mathrm{p}<0.001)$ vending snacks. There were also significant time by condition effects for vending snacks (Table 4). The proportion of Do Not Sell snacks in GL + CBI facilities declined from $74.0 \pm 16.6 \%$ to $45.2 \pm 20.1 \%$ compared to GLONLY $(80.0 \pm 22.4 \%$ to $81.0 \pm 22.1 \% ; \mathrm{p}<0.01)$ and NO-GL facilities $(91.1 \pm 8.8 \%$ to $90.6 \pm 6.3 \%$; $p<0.001)$. The proportion of Sell Sometimes snacks in GL + CBI facilities increased from $22.4 \pm 14.4 \%$ to $43.8 \pm 15.8 \%$ compared to GL-ONLY $(15.0 \pm 15.2 \%$ to $15.2 \pm 16.6 \% ; p<0.01)$ and NOGL facilities $(6.0 \pm 6.3 \%$ to $6.7 \pm 5.1 \%$; $p<0.001)$. For Sell Most snacks the overall time by condition effect was significant $(p=0.002)$, however post-hoc analyses revealed no significant pairwise differences, although there was a trend toward an increase in the proportion of Sell Most snacks in $\mathrm{GL}+\mathrm{CBI}(3.7 \pm 4.4 \%$ to $11.0 \pm 9.0 \%)$ relative to NO-GL facilities (2.8 \pm 3.4 to $2.7 \pm 4.7 ; p=0.08)$.

\section{Food environment - concessions}

There were no significant effects of time or time by condition for packaged snacks and beverages in concessions (Table 5).

Table 1 Capacity building intervention (CBI) elements for the Eat, Play, Live trial

\begin{tabular}{|c|c|c|}
\hline CBI resources and supports & Frequency and timing & Description \\
\hline \multirow[t]{6}{*}{ Province-specific training } & \multirow[t]{6}{*}{ Once, 0 months } & $\begin{array}{l}\text { Half-day, in-person group training covering the following: } \\
\text { - Five-step change process }\end{array}$ \\
\hline & & $\begin{array}{l}\text { - Operational areas for policy and program changes in } \\
\text { food environments }\end{array}$ \\
\hline & & $\begin{array}{l}\text { - Practical examples of food environment changes for } \\
\text { each operational area }\end{array}$ \\
\hline & & - Provincial nutrition guidelines \\
\hline & & $\begin{array}{l}\text { - Resources to support food environment and policy } \\
\text { change (see below) }\end{array}$ \\
\hline & & - CBI protocol and timeline \\
\hline Baseline evaluation report & Once, 0 months & $\begin{array}{l}\text { All facilities received a report summarizing data collected } \\
\text { at baseline in their facility on facility capacity and food } \\
\text { environment quality }\end{array}$ \\
\hline Seed funds & Once, 3 months & $\$ 1000$ CAD per facility \\
\hline Online toolkit & Unlimited access & $\begin{array}{l}\text { BC Recreation and Parks Association website } \\
\text { (stayactiveeathealthy.ca) provides information on healthy } \\
\text { food in recreation. Includes searchable database of } \\
\text { resources and provincial pages with local resources }\end{array}$ \\
\hline Online resources & Unlimited access & $\begin{array}{l}\text { Local resource hubs to support environment and policy } \\
\text { change were provided in BC (https://bnfl.healthlinkbc.ca/) } \\
\text { AB (healthyeatingstartshere.ca; apccprecproject.com) and } \\
\text { NS (https://thrive.novascotia.ca/, https://thrive.novascotia. } \\
\text { ca/resources) }\end{array}$ \\
\hline $\begin{array}{l}\text { Provincial Coordinator } \\
\text { support }\end{array}$ & Monthly follow-up and as needed & $\begin{array}{l}\text { On-demand individual training, resources, and support } \\
\text { (e.g. assistance with nutrition analysis, product sourcing, } \\
\text { connecting with community resources including public } \\
\text { health dietitians) via email, telephone and in-person }\end{array}$ \\
\hline Provincial teleconferences & $2,6,12$ months & $\begin{array}{l}60 \text { min intra-provincial cross-facility meetings; facilities de- } \\
\text { scribed current and planned activities, challenges and } \\
\text { facilitators }\end{array}$ \\
\hline Facility action plans & $0,3,6,12,18$ months & $\begin{array}{l}\text { CBI facilities developed written implementation plans to } \\
\text { achieve their goals }\end{array}$ \\
\hline $\begin{array}{l}\text { Electronic information } \\
\text { provision }\end{array}$ & Every $1-2$ months & $\begin{array}{l}\text { Periodic group emails from Provincial Coordinators } \\
\text { addressing common inquiries, challenges, and successes }\end{array}$ \\
\hline
\end{tabular}


Table 2 Facility characteristics at baseline for the Eat, Play, Live trial

\begin{tabular}{|c|c|c|c|}
\hline Characteristic & $\begin{array}{l}\text { NO-GL } \\
n=17\end{array}$ & $\begin{array}{l}\text { GL-ONLY } \\
n=15\end{array}$ & $\begin{array}{l}\mathrm{GL}+\mathrm{CBI} \\
n=17\end{array}$ \\
\hline \multicolumn{4}{|l|}{ Number of facilities $(n)$} \\
\hline British Columbia & 0 & 7 & 7 \\
\hline Alberta & 0 & 5 & 6 \\
\hline Nova Scotia & 0 & 3 & 4 \\
\hline Ontario & 17 & 0 & 0 \\
\hline \multicolumn{4}{|l|}{ Community size (\% of facilities) ${ }^{a}$} \\
\hline Rural & 0 & 6.7 & 5.9 \\
\hline Small population centre & 17.6 & 66.0 & 47.0 \\
\hline Medium population centre & 17.6 & 0 & 5.9 \\
\hline Large urban population centre & 64.7 & 26.7 & 41.2 \\
\hline \multicolumn{4}{|l|}{ Facility size ( $\%$ of facilities $)^{b}$} \\
\hline Small & 5.9 & 46.7 & 35.3 \\
\hline Medium & 35.3 & 20.0 & 23.5 \\
\hline Large & 58.8 & 33.3 & 41.2 \\
\hline Mean number of vending machines (range) & $6.5(1-15)$ & $3.6(0-11)$ & $5.8(0-25)$ \\
\hline Mean number of concessions (range) & $1.1(1-2)$ & $0.8(0-2)$ & $1.1(0-4)$ \\
\hline
\end{tabular}

$G L+C B I$ guideline + capacity building intervention facilities, GL-ONLY guideline only facilities, NO-GL no guideline facilities

avalues obtained from Statistics Canada [66] and classified according to Statistics Canada definitions [67]

${ }^{\mathrm{b}} \mathrm{A}$ small facility had at least one minor or major amenity; a medium facility had at least two amenities, one of which was major; a large facility had at least three amenities, two of which were major. Major amenities: ice rink, swimming pool, multiple courts (e.g. tennis, squash/racquetball), bowling alley, theatre (e.g. movie, performing arts). Minor amenities: community and multi-purpose room, fitness room, small specialty area such as small climbing wall or gymnastics room

There were no significant effects of time for rNEMS-R scores. There were significant time by condition effects for the fast food version of the rNEMS-R score (Table 5). rNEMS-R scores increased in GL + CBI $(14.7 \pm 8.4$ to $17.5 \pm 7.2)$, compared to NO-GL facilities $(9.2 \pm 4.3$ to $4.0 \pm 4.4 ; \mathrm{p}<0.001)$, and declined in NO-GL $(9.2 \pm 4.3$ to $4.0 \pm 4.4)$ relative to GL-ONLY facilities $(14.8 \pm 6.5 \%$ to $14.1 \pm 5.1 ; p<0.01)$. These changes trended in a similar direction using the fast casual version of the rNEMS-R score, but were not statistically significant $(\mathrm{p}=0.08)$.

GL + CBI facilities exhibited the healthiest mix of marker foods over time. Nearly three-quarters increased/maintained provision of fruits and vegetables, nearly half offered a healthier mix of potato chips (i.e. more baked and fewer regular varieties) and more than one-third increased/maintained provision of healthy main and side dishes (Table 6).

\section{Sensitivity analyses}

Findings were unchanged in sensitivity analyses except in one respect, as there was a significant effect of time by condition for rNEMS-R fast casual scores. rNEMS-R scores increased in GL + CBI $(13.8 \pm 8.0 \%$ to $16.7 \pm 6.1 \%)$ compared to NO-GL facilities $(8.4 \pm 3.5$ to $7.3 \pm 3.8 ; \mathrm{p}<$ 0.01 ), and contrary to expectations declined in GL-
ONLY $(15.5 \pm 8.0$ to $13.2 \pm 5.3)$ relative to NO-GL facilities $(8.4 \pm 3.5$ to $7.3 \pm 3.8 ; \mathrm{p}<0.01)$.

\section{Discussion}

The EPL trial leveraged differences in provincial nutrition guidelines for recreation and sport facilities as an opportunity to examine the impact of an 18 month CBI in enhancing implementation of nutrition guidelines. We also examined whether nutrition guidelines were associated with positive changes over time. Primary study outcomes were facility capacity, policy development and food environment quality. Although facilities in guideline provinces started at a healthier place relative to those in a province without nutrition guidelines (unpublished data), provincial nutrition guidelines alone did not provoke positive changes during this 18 month study. Significant improvements in facility capacity, policy development, and in food environment quality (vending and concession) only occurred in facilities that were both exposed to nutrition guidelines and participated in an actively delivered CBI. Thus, ongoing capacity building is needed to realize the positive potential of voluntary nutrition guidelines. Nevertheless, food environments remained overwhelmingly unhealthy in all cases, suggesting additional scope to enhance implementation of nutrition guidelines. 
Table 3 Change in facility capacity by intervention group in the Eat, Play, Live trial

\begin{tabular}{|c|c|c|c|c|c|}
\hline & \multirow[t]{2}{*}{$n$} & \multicolumn{2}{|c|}{ Mean \% ideal score (SD) } & \multicolumn{2}{|c|}{ Intervention effect ${ }^{a}$} \\
\hline & & Baseline & Follow-up & $\mathrm{F}$ & $p$ value \\
\hline \multicolumn{6}{|c|}{ Facility capacity subscores } \\
\hline \multicolumn{6}{|c|}{ Strategic planning } \\
\hline $\mathrm{GL}+\mathrm{CBI}$ & 16 & $22.9(24.1)$ & $60.9(28.2)$ & 10.050 & $<0.001^{\mathrm{b}}$ \\
\hline GL only & 13 & $16.0(26.2)$ & $16.0(25.8)$ & & \\
\hline No GL & 14 & $6.0(12.4)$ & $23.2(23.6)$ & & \\
\hline \multicolumn{6}{|c|}{ Communication and education } \\
\hline $\mathrm{GL}+\mathrm{CBI}$ & 16 & $36.1(19.8)$ & $63.2(27.1)$ & 3.890 & $0.029^{c}$ \\
\hline GL only & 13 & $29.1(23.7)$ & $30.8(28.1)$ & & \\
\hline No GL & 14 & $21.8(15.0)$ & $40.1(19.1)$ & & \\
\hline \multicolumn{6}{|c|}{ Overall facility capacity score } \\
\hline \multicolumn{6}{|l|}{ Total } \\
\hline $\mathrm{GL}+\mathrm{CBI}$ & 16 & $30.8(15.6)$ & $62.3(22.0)$ & 7.908 & $0.001^{b}$ \\
\hline GL only & 13 & $23.8(21.7)$ & $24.9(26.1)$ & & \\
\hline No GL & 14 & $15.5(12.9)$ & $33.3(14.3)$ & & \\
\hline
\end{tabular}

$G L+C B I$ guideline + capacity building intervention facilities, $G L$ only guideline only facilities, No GL no guideline facilities

At follow-up $1 \mathrm{GL}+\mathrm{CBI}, 2 \mathrm{GL}-\mathrm{ONLY}$ and 3 NO-GL facilities did not report facility capacity scores

aThe intervention effect is the $p$ value for the interaction of time by condition from repeated measures ANOVA

${ }^{\mathrm{b}}$ Significant pairwise differences between $\mathrm{GL}+\mathrm{CBI}$ and GL-ONLY $(p<0.01)$; and between $\mathrm{GL}+\mathrm{CBI}$ and NO-GL $(p<0.01)$

'Significant pairwise differences between GL + CBI and GL-ONLY $(p<0.05)$; and between $\mathrm{GL}+\mathrm{CBI}$ and NO-GL $(p<0.05)$

Effective nutrition policies have been defined as those that lead to positive changes in food, social and informational environments [35]. Improvements to food environments may, in turn, improve children's dietary intake $[5,6]$. To be effective in changing food environments and dietary intake however, policies must be implemented. The difficulty of achieving high levels of policy implementation can be substantial [36-39], particularly when policies are voluntary [2, 17]. Indeed, although guideline facilities had healthier food environments than no guideline facilities at baseline (unpublished observations), their food environments remained overwhelmingly unhealthy, demonstrating a failure to fully implement nutrition guidelines. Moreover, there were no positive changes in the food environments of guideline only facilities over the course of the study. This suggests that provincial nutrition guidelines may have prompted early positive change in recreation and sport facility food environments, but that without ongoing support, the change process stagnated. It is possible that managers perceived they were doing enough, and that further change was not needed. Having mandated policies, with clear standards of accountability, could help to ensure managers understand
Table 4 Change in the proportion of snacks and beverages in vending machines by intervention group in the Eat, Play, Live trial

\begin{tabular}{|c|c|c|c|c|c|}
\hline & \multirow[t]{2}{*}{$n$} & \multicolumn{2}{|c|}{ Mean \% (SD) } & \multicolumn{2}{|c|}{ Intervention effect ${ }^{a}$} \\
\hline & & Baseline & Follow-up & $\mathrm{F}$ & $p$ value \\
\hline \multicolumn{6}{|l|}{ Beverages } \\
\hline \multicolumn{6}{|l|}{ Do Not Sell } \\
\hline $\mathrm{GL}+\mathrm{CBI}$ & 16 & $59.1(17.2)$ & $51.8(20.1)$ & 0.667 & 0.52 \\
\hline GL-ONLY & 12 & $71.7(14.1)$ & $64.8(20.5)$ & & \\
\hline NO-GL & 17 & $80.8(10.2)$ & $79.2(14.4)$ & & \\
\hline \multicolumn{6}{|c|}{ Sell Sometimes } \\
\hline $\mathrm{GL}+\mathrm{CBI}$ & 16 & $21.4(12.7)$ & $24.9(16.2)$ & 0.043 & 0.96 \\
\hline GL-ONLY & 12 & $14.6(12.6)$ & $18.7(13.2)$ & & \\
\hline NO-GL & 17 & $10.8(4.4)$ & $13.4(9.2)$ & & \\
\hline \multicolumn{6}{|l|}{ Sell Most } \\
\hline $\mathrm{GL}+\mathrm{CBI}$ & 16 & $19.4(11.9)$ & $23.3(11.3)$ & 1.596 & 0.22 \\
\hline GL-ONLY & 12 & $13.7(7.4)$ & $16.5(11.3)$ & & \\
\hline NO-GL & 17 & $8.5(9.8)$ & $7.4(9.1)$ & & \\
\hline \multicolumn{6}{|l|}{ Snacks } \\
\hline \multicolumn{6}{|l|}{ Do Not Sell } \\
\hline $\mathrm{GL}+\mathrm{CBI}$ & 14 & $74.0(16.6)$ & $45.2(20.1)$ & 15.629 & $<0.001^{b}$ \\
\hline GL-ONLY & 10 & $80.0(22.4)$ & $81.0(22.1)$ & & \\
\hline NO-GL & 12 & $91.1(8.8)$ & $90.6(6.3)$ & & \\
\hline \multicolumn{6}{|c|}{ Sell Sometimes } \\
\hline $\mathrm{GL}+\mathrm{CBI}$ & 14 & $22.4(14.4)$ & $43.8(15.8)$ & 15.529 & $<0.001^{\mathrm{b}}$ \\
\hline GL-ONLY & 10 & $15.0(15.2)$ & $15.2(16.6)$ & & \\
\hline NO-GL & 12 & $6.0(6.3)$ & $6.7(5.1)$ & & \\
\hline \multicolumn{6}{|l|}{ Sell Most } \\
\hline $\mathrm{GL}+\mathrm{CBI}$ & 14 & $3.7(4.4)$ & $11.0(9.0)$ & 4.310 & $0.002^{c}$ \\
\hline GL-ONLY & 10 & $4.9(8.3)$ & $3.8(6.5)$ & & \\
\hline NO-GL & 12 & $2.8(3.4)$ & $2.7(4.7)$ & & \\
\hline
\end{tabular}

$\overline{G L}+C B I$ guideline + capacity building intervention facilities, GL-ONLY guideline only facilities, NO-GL no guideline facilities

A total of 83 beverage ( $52 \%$ of all beverage machines; $n=2110$ products) and 58 snack vending machines ( $78 \%$ of all snack machines; $n=1983$ products) were audited at baseline. A total of 79 beverage ( $54 \%$ of all beverage machines; $n=2106$ products) and 59 snack vending machines ( $80 \%$ of all snack machines; $n=2067$ products) were audited at follow-up

${ }^{a}$ The intervention effect is the $p$ value for the interaction of time by condition from repeated measures ANOVA

${ }^{\mathrm{b}}$ Significant pairwise differences between GL $+\mathrm{CBI}$ and GL-ONLY $(p<0.01)$; and between $\mathrm{GL}+\mathrm{CBI}$ and NO-GL $(\mathrm{p}<0.001)$

${ }^{c}$ Non-significant trend toward pairwise differences between $\mathrm{GL}+\mathrm{CBI}$ and NO-GL $(p=0.08)$

what constitutes meaningful change to improve food environments.

Organizational capacity building enhanced implementation of voluntary provincial nutrition guidelines in recreation and sport facilities across three provinces. These changes were realized at a relatively low cost of approximately $\$ 2950 /$ facility (CAD). The most 
Table 5 Change in the proportion of packaged snacks and beverages and in the overall quality of the concession food environment by intervention group in the Eat, Play, Live trial

\begin{tabular}{llllll}
\hline & $n$ & Mean \% (SD) & & Intervention effect \\
& Baseline & Follow-up & & F value \\
\hline Packaged beverages & & & &
\end{tabular}

Packaged beverages

Do Not Sell

$\mathrm{GL}+\mathrm{CBI}$

$9 \quad 73.1(9.4)$

$74.2(9.5)$

0.478

0.62

GL-ONLY

1171.8 (13.6)

$73.7(9.8)$

NO-GL

$1680.3(9.2)$

$85.3(8.4)$

Sell Sometimes

$\begin{array}{lllll}\mathrm{GL}+\mathrm{CBI} & 9 & 19.0(10.6) & 20.3(10.7) & 0.89 \\ \text { GL-ONLY } & 11 & 20.6(15.9) & 18.2(11.2) \\ \text { NO-GL } & 16 & 16.6(8.4) & 11.8(6.9)\end{array}$

Sell Most

$\mathrm{GL}+\mathrm{CBI}$

$9 \quad 7.9(3.5)$

5.5 (2.9)

1.805

GL-ONLY

$117.6(6.5)$

$8.1(8.8)$

NO-GL

$16 \quad 3.2(3.3)$

$2.9(3.9)$

Packaged snacks

Do Not Sell

$\mathrm{GL}+\mathrm{CBI}$

GL-ONLY

NO-GL

$7 \quad 83.4(18.3)$

$69.1(21.6)$

1.550

0.23

$11 \quad 74.9(25.6) \quad 78.0(12.4)$

Sell Sometimes

GL+CBI

$16 \quad 93.6(7.1)$

$93.7(9.3)$

Sell Most

$\mathrm{GL}+\mathrm{CBI}$
$\mathrm{GL}-\mathrm{ONLY}$

$7 \quad 15.1(17.8)$

$1121.0(27.4)$

$165.6(6.3)$

$26.5(16.9)$

1.213

0.31

$17.9(9.8)$

$5.4(9.2)$

EMS-R Scores ${ }^{a}$

\begin{tabular}{|c|c|c|c|c|c|}
\hline \multirow[b]{2}{*}{ Fast casual score } & & \multicolumn{2}{|l|}{$\begin{array}{l}\text { Mean (SD) } \\
\text { [\% ideal] }\end{array}$} & \multicolumn{2}{|c|}{ Intervention effect ${ }^{b}$} \\
\hline & & Baseline & Follow-up & $\mathrm{F}$ & $p$ value \\
\hline \multirow[t]{2}{*}{$\mathrm{GL}+\mathrm{CBI}$} & 11 & $15.5(9.0)$ & $17.7(6.7)$ & 2.675 & 0.08 \\
\hline & & {$[37.3 \%]$} & {$[41.5 \%]$} & & \\
\hline \multirow[t]{2}{*}{ GL-ONLY } & 11 & $15.5(8.0)$ & $13.2(5.3)$ & & \\
\hline & & {$[37.3 \%]$} & [32.9\%] & & \\
\hline \multirow[t]{2}{*}{ NO-GL } & 16 & $8.4(3.5)$ & $7.3(3.8)$ & & \\
\hline & & {$[23.8 \%]$} & [21.6\%] & & \\
\hline \multicolumn{6}{|l|}{ Fast food score } \\
\hline \multirow[t]{2}{*}{$\mathrm{GL}+\mathrm{CBI}$} & 11 & $14.7(8.4)$ & $17.5(7.2)$ & 10.958 & $<0.001^{c}$ \\
\hline & & {$[40.4 \%]$} & [45.0\%] & & \\
\hline \multirow[t]{2}{*}{ GL-ONLY } & 11 & $14.8(6.5)$ & $14.1(5.1)$ & & \\
\hline & & [40.6\%] & [39.4\%] & & \\
\hline
\end{tabular}

Table 5 Change in the proportion of packaged snacks and beverages and in the overall quality of the concession food environment by intervention group in the Eat, Play, Live trial (Continued)

\begin{tabular}{|c|c|c|c|c|}
\hline & \multirow[t]{2}{*}{$n$} & \multicolumn{2}{|c|}{ Mean \% (SD) } & Intervention effect $^{a}$ \\
\hline & & Baseline & Follow-up & $p$ value \\
\hline \multirow[t]{2}{*}{ NO-GL } & 16 & $9.2(4.3)$ & $4.0(4.4)$ & \\
\hline & & [31.5\%] & {$[23.0 \%]$} & \\
\hline
\end{tabular}

$\overline{G L+C B I \text { guideline + capacity building intervention facilities, GL-ONLY guideline }}$ only facilities, NO-GL no guideline facilities, rNEMS-R Nutrition Environment Measures Survey-Restaurant reduced item audit

A total of 40 concessions were audited at baseline and 38 concessions were audited at follow-up. 8 facilities did not have a concession, 1 could not be audited at baseline or follow-up and 2 concessions closed between baseline and follow-up. In addition, 1 concession no longer sold packaged beverages and 2 no longer sold packaged snacks at follow-up. A total of 756 packaged beverages and 772 packaged snacks were audited at follow-up

aHigher scores (possible range for fast casual: -4.02 to +48.27 ; fast food:

-10.17 to +51.35 ) indicate greater availability, supports for, and lower cost of healthy options

${ }^{b}$ The intervention effect is the $p$ value for the interaction of time by condition from repeated measures ANOVA

'Significant pairwise differences between $\mathrm{GL}+\mathrm{CBI}$ and NO-GL $(p<0.001)$; and between GL-ONLY and NO-GL $(p<0.01)$

significant changes observed were with respect to the quality of snack provision within vending machines, with significant declines in unhealthy, and significant increases in healthier products in GL + CBI facilities. In a pilot study of an early version of the CBI we also observed positive shifts in the provision of vending products over a shorter 8 month time frame [2]. Although results for concession snacks trended in similar directions, the variability among facilities was high and thus there were no significant changes. Given that some facilities did not have concessions, this analysis may also have been underpowered. A nearly $5 \%$ improvement in the overall quality of concession food environments was, however, observed in GL + CBI facilities, along with positive shifts in the proportion of facilities offering healthy marker foods, including fruits, vegetables, healthy main dishes and sides. The latter findings may be more informative than changes in the proportional availability of healthy and unhealthy concession items, as items sold in concessions are not typically prominently displayed as they are in vending machines (i.e. most items are simply listed in text on menu boards and/ or a single facing of each item is on display). As such the proportionality metric may be a less informative indicator of food environment quality in concessions. The only other RCT conducted in a similar context also found positive shifts in the provision of fruits and vegetables following a multi-component canteen intervention, albeit on the basis of self-reported data [40]. However, in agreement with our findings, there were no significant changes in availability of non-sugar-sweetened drinks. It is possible that managers were attempting to limit their risk through step-wise changes and perceived greater opportunity to improve the healthfulness of meals and snacks. 
Table 6 Availability of marker foods and food preparation equipment in the Eat, Play, Live trial

\begin{tabular}{|c|c|c|c|c|}
\hline & \multicolumn{3}{|c|}{ Proportion of facilities that made healthy changes or maintained a healthy status from baseline to follow-up } & \multirow[t]{2}{*}{$p$ value } \\
\hline & $\begin{array}{l}\overline{G L}+C B I \\
(n=11)\end{array}$ & $\begin{array}{l}\text { GL-ONLY } \\
(n=11)\end{array}$ & $\begin{array}{l}\text { NO-GL } \\
(n=16)\end{array}$ & \\
\hline \multicolumn{5}{|l|}{ Foods and beverages } \\
\hline Low fat milk & $9.1 \%$ & $18.2 \%$ & $0 \%$ & 0.22 \\
\hline 100\% juice & $90.9 \%$ & $100 \%$ & $100 \%$ & 0.28 \\
\hline Whole grain bread & $72.7 \%$ & $63.6 \%$ & $37.5 \%$ & 0.16 \\
\hline White bread & $9.1 \%$ & $9.1 \%$ & $18.8 \%$ & 0.69 \\
\hline French fries & $36.4 \%$ & $45.5 \%$ & $31.3 \%$ & 0.75 \\
\hline Potato chips ${ }^{a}$ & $45.5 \%$ & $27.3 \%$ & $0 \%$ & 0.015 \\
\hline Fruit & $72.7 \%$ & $81.8 \%$ & $12.5 \%$ & $<0.001$ \\
\hline Vegetables & $72.7 \%$ & $36.4 \%$ & $6.3 \%$ & 0.002 \\
\hline High fat side & $27.3 \%$ & $18.2 \%$ & $25.0 \%$ & 0.87 \\
\hline Healthy main dish & $36.4 \%$ & $9.1 \%$ & $0 \%$ & 0.021 \\
\hline Healthy main dish salad & $36.4 \%$ & $9.1 \%$ & $0 \%$ & 0.021 \\
\hline \multicolumn{5}{|l|}{ Food preparation equipment } \\
\hline Deep fat fryer & $36.4 \%$ & $45.5 \%$ & $31.3 \%$ & 0.75 \\
\hline Griddle & $45.5 \%$ & $45.5 \%$ & $81.3 \%$ & 0.08 \\
\hline Grill & $45.5 \%$ & $9.1 \%$ & $18.8 \%$ & 0.11 \\
\hline Oven & $63.6 \%$ & $45.5 \%$ & $56.3 \%$ & 0.69 \\
\hline
\end{tabular}

$G L+C B I$ guideline + capacity building intervention facilities, GL-ONLY guideline only facilities, NO-GL no guideline facilities, $r N E M S-R$ reduced Nutrition Environment Measures Survey - Restaurant

Healthy change/maintenance scores were calculated using Chi-square statistics based on the presence or absence of the item at baseline and follow-up (i.e. a healthy change was not selling a healthy item at baseline but selling it at follow-up or selling an unhealthy item at baseline and not selling it at follow-up; maintenance of a healthy status was selling a healthy item at baseline and follow-up)

A total of 40 concessions were audited at baseline and 38 concessions were audited at follow-up. 8 facilities did not have a concession, 1 could not be audited at baseline or follow-up and 2 concessions closed between baseline and follow-up

${ }^{a}$ Potato chips combines information about the change in availability of baked and regular potato chips from baseline to follow-up. For facilities that sold regular and baked, or only baked potato chips at baseline, a healthy change was defined as no longer selling any potato chips at follow-up. For facilities that sold only regular potato chips at baseline a healthy change was defined as no longer selling regular chips and/or adding baked chips at follow-up. Selling baked chips at baseline and follow-up was classified as maintaining a healthy status

Organizational capacity building is an evidence-based strategy that can support organizations in mobilizing policy into practice by ensuring adequate resources, supportive structures and workforce skills are available [41]. The EPL CBI succeeded in building facility capacity, as evidenced by significant increases in capacity scores in GL + CBI facilities (from 31 to $62 \%$ of ideal score). These increases in facility capacity coincided with significant increases in nutrition policy development at a facility level and in the quality of food environments. Although smaller, but non-significant increases in capacity were also observed in NO-GL facilities (from 16 to $33 \%$ of ideal score), there were no concurrent changes in policy development or food environment quality. This may suggest a threshold effect. NO-GL facilities may not have reached the critical capacity threshold capable of yielding significant positive change. Our findings clearly show that one reason why provincial nutrition guidelines have not been fully implemented in recreation and sport facilities is because facilities lack capacity to do so. That scores were just $62 \%$ of ideal in GL + CBI facilities suggests there may be additional scope to improve food environments through further capacity building. Alternatively, that large changes in facility capacity did not translate into larger positive changes in food environments may also indicate that capacity building alone is not sufficient to create truly healthy food environments in the context of voluntary nutrition guidelines, and where cultural norms promote the opposite. A multi-component implementation intervention in Australian sports clubs that included several analogous implementation strategies also found positive, albeit limited improvements in food environments [42].

Policy-focused and policy-supported interventions have proven effective in improving population health [43-46] and therefore the CBI encouraged development of nutrition policies at a facility level. Whereas no comparison facilities developed written nutrition policies during the course of the study, nearly one-fifth of GL + CBI facilities did. The deliberative processes involved in 
formalizing written policies can assist facilities to clarify and formalize their core values [47], and may therefore have stimulated change to food environments within these facilities. Moreover, policies codify change and often survive transitions in leadership, which may enhance the sustainability of change [48]. The presence of facility-specific nutrition policies at baseline was associated with healthier food environments (unpublished observations). We therefore expect that provincial and local policies might be mutually reinforcing over time.

Despite significant improvements in some aspects of facilities' food environments, they nevertheless remained overwhelmingly unhealthy. Several factors in combination may explain these findings. First, change takes time. Unhealthy food and drink are deeply embedded within the culture of sport $[4,19]$, and change will require both initiative-taking on the part of mangers to supply healthier foods, and a demand 'pull' from consumers to purchase these items. As gate-keepers of food environments in recreation and sport, managers are crucial to realizing such change. Through incrementally improving the nutritional quality of foods on offer, managers can help to gradually accustom patrons to seeing and purchasing healthier foods [4]. Second, although the CBI addressed important barriers previously identified in this context (e.g. perceived lower profitability of healthier items, limited knowledge and skills to implement nutrition guidelines, minimal stakeholder buy-in and cooperation $[1,2,4,18,19])$, the strategies presented in the CBI may not have been sufficient to fully overcome them. Moreover, the CBI did not address barriers at the patron level, as parents admit to purchasing unhealthy foods for their children because factors such as convenience, cost and child preferences sometimes take precedence over health [49-52]. More than 80 distinct capacity building elements have been identified in the literature [53]. Thus, greater tailoring of the CBI to the needs and contexts of particular facilities may also be required [54]. Third, EPL focussed on building capacity at the organizational rather than at the individual practitioner level, and these two types of capacity are mutually reinforcing [41]. Building capacity at both levels may provoke greater change. Finally, low availability of healthy, shelf-stable, tasty products in the marketplace likely limited the extent of change that facilities could make [18].

\section{Implications}

Unhealthy food environments may promote correspondingly unhealthy dietary patterns, particularly among children who have less control over their surroundings [5]. It is therefore concerning that the majority of recreation and sport facilities had unhealthy food environments regardless of the presence of voluntary nutrition guidelines. This is consistent with our previous findings in recreation facilities in Canada $[1,2,4$, $16]$, and with studies internationally in a variety of sports settings [40, 55-59]. As identified in a recent systematic review, there is almost no knowledge of how to improve policy implementation in sports settings, with just three controlled studies meeting inclusion criteria [60]. EPL demonstrated how principles related to organizational capacity building can be successfully operationalized to improve implementation of nutrition guidelines in recreation and sport facilities at a relatively low cost. Findings provide key data for policy makers seeking to enhance implementation of voluntary guidelines, which may be more politically palatable than mandated policy. Even the small changes observed here can support healthier dietary patterns at a population-level, and may portend greater benefits in future by initiating processes of change. Dietary patterns established in childhood and adolescence may be enduring [61, 62], and thus reengineering food environments in recreation and sport facilities remains a high priority. Ultimately, mandated, monitored and enforced nutrition policies, alongside change in social norms, may be required to achieve full implementation of nutrition guidelines.

\section{Strengths and limitations}

The EPL trial was designed to maximize both internal and external validity by embedding a RCT within a natural experiment conducted across provinces at different stages of guideline implementation. Given the heterogeneity in provincial contexts, stages of guideline implementation, facility types and sizes, and the remarkable consistency in the barriers to change encountered in previous Canadian [2, 4, 17-19, 63] and international studies [52, 64, 65], we expect the generalizability of findings to be relatively high. Nevertheless, the poor representation of rural and medium population centres remains a limitation.

The CBI had a strong theoretical grounding in a linking system approach. It incorporated evidence-based elements identified in the wider capacity-building literature and also targeted specific barriers previously encountered in this setting. It also mimicked a real-world context by relying largely on existing supports and resources to support recreation stakeholders in the change process, and by having facilities create and execute their own contextualized action plans. These features may enhance the sustainability of the changes observed here, however, we do not have data in this respect. Ultimately, the sustainability of change will likely depend on whether and to what extent any changes influence profitability. 
Risk of bias was minimized by randomization, objective assessment of food environments by trained Research Assistants, and by the lack of attrition and little missing data. However, limitations remain, as food environments were assessed at a single point in time, although previous data indicate that the contents of vending machines do not typically vary substantially from week to week [24]. It was not possible to comprehensively measure all aspects of food environments, and thus facilities may have made changes in other areas that were not captured here. This could have had unintended consequences, whereby managers focussed on making changes in the areas we assessed, while letting others go unaddressed. Our analyses were underpowered to detect small changes, thereby increasing the chance of type 2 errors. With a larger sample size, some non-significant trends in the data may have reached statistical significance. Guideline facilities were randomly assigned to GL + CBI or GL-ONLY conditions, however facilities could not be randomly assigned to the NO-GL condition. However, as all facilities were publicly funded, they did not self-select to locate in a particular province and therefore assignment to condition occurred through what was essentially a random process. It is possible that facilities with a greater interest in nutrition may have been more likely to participate in the study. Finally, the impact of changes to food environments on children's food intake was not assessed.

\section{Conclusions}

Findings from the EPL trial show the potential to leverage a capacity building approach that relies largely on existing resources and supports to protect government investments in developing and disseminating voluntary nutrition guidelines for recreation and sport facilities. Significant improvements in facility capacity, policy development and food environment quality occurred in recreation and sport facilities that were both exposed to nutrition guidelines and that participated in an actively delivered CBI. Outcomes did not improve significantly in facilities that were only passively or not at all exposed to provincial nutrition guidelines. Although facilities in guideline provinces started at a healthier place relative to those in a province without nutrition guidelines, without ongoing capacity building the change process ultimately stagnated. Food environments remained overwhelmingly unhealthy in all facilities, however, suggesting additional scope to enhance implementation of nutrition guidelines through more extensive capacity building. Alternatively, mandated, monitored and enforced nutrition policies may be required to ensure that food environments in recreation and sport facilities support, and do not undermine, children's health.

\section{ADDITIONAL FILES}

Additional file 1: CONSORT checklist. (PDF $147 \mathrm{~kb}$ )

Additional file 2: TIDieR checklist. (PDF $206 \mathrm{~kb}$ )

Additional file 3: Facilities Assessment questionnaire. (PDF 219 kb)

Additional file 4: Vending audit. (PDF 390 kb)

Additional file 5: Nutrition Environment Measures Survey-Restaurant reduced item audit. (PDF $311 \mathrm{~kb}$ )

\section{Abbreviations}

AB: Alberta; BC: British Columbia; CBI: Capacity building intervention; EPL: Eat, Play, Live; GL + CBI: Guideline + capacity building intervention; GL-

ONLY: Guideline only; NEMS-R: Nutrition Environment Measures SurveyRestaurant; NO-GL: No guideline; NS: Nova Scotia; RCT: Randomized controlled trial; rNEMS-R: Nutrition Environment Measures Survey-Restaurant reduced item audit

\section{Acknowledgements}

We acknowledge the students and staff who acted as Research Coordinators and Assistants and assisted with data collection and entry, along with participating facility managers, staff and vendors; EPL could not have been carried out without their willing participation. We are also grateful to Yun Yun Lee for her excellent assistance in preparing tables and figures for this manuscript.

\section{Authors' contributions}

DLO, KDR, SK, JLM, RH, LCM, PJN: designed the study and obtained funding; RJLP, DT, MSC: collected the data; DT, TM, PJN: analysed the data; DLO, RJLP, DT: developed tables and figures; DLO: wrote the manuscript. All authors interpreted the data, read, edited and approved the final manuscript.

\section{Funding}

This study was funded by the Heart and Stroke Foundation of Canada. The study funder had no role in the design of the study, in the collection, analysis and interpretation of the data, or in writing the manuscript. LCM received salary support from the BC Children's Hospital Research Institute.

\section{Availability of data and materials}

The datasets used and analysed during the current study are available from the corresponding author on reasonable request.

\section{Ethics approval and consent to participate}

The study received ethical approval from the Health Research Ethics Boards at the University of Victoria, the University of British Columbia, the University of Alberta, the University of Waterloo and Dalhousie University. Managers of recreation facilities agreed to their facilities' participation.

\section{Consent for publication}

Not applicable.

\section{Competing interests}

DLO and KDR were members of the committee that generated a draft version of the Alberta Nutrition Guidelines for Children and Youth. All other authors declare that they have no competing interests.

\section{Author details}

'Department of Community Health Sciences, Cumming School of Medicine, University of Calgary, 3280 Hospital Drive NW, Calgary, AB T2N 4Z6, Canada. ${ }^{2}$ School of Public Health, University of Alberta, 1140587 Avenue NW, Edmonton, AB T6G 1C9, Canada. ${ }^{3}$ School of Exercise Science, Physical and Health Education, University of Victoria, PO Box 3015 Stn CSC, Victoria, BC V8W 3P1, Canada. ${ }^{4}$ Healthy Populations Institute, Dalhousie University, Stairs House, PO Box 15000, 6230, South Street, Halifax, NS B3H 4R2, Canada.

${ }^{5}$ Faculty of Education and Department of Child and Youth Study, Mount Saint Vincent University, 166 Bedford Hwy, Halifax, NS B3M 2J6, Canada. ${ }^{6}$ BC Children's Hospital Research Institute, School of Population and Public Health, University of British Columbia, 4480 Oak Street, Vancouver, BC V6H 3V4, Canada. ${ }^{7}$ School of Public Health and Health Systems, University of Waterloo, 200 University Ave West, Waterloo, ON N2L 3G1, Canada. ${ }^{8}$ Department of Curriculum and Instruction, Faculty of Education, University of Victoria, PO Box 1700, STN CSC, Victoria, BC V8W 2YW, Canada. 
Received: 22 November 2018 Accepted: 5 June 2019

Published online: 25 June 2019

\section{References}

1. Naylor PJ, Bridgewater L, Purcell M, Ostry A, Wekken SV. Publically funded recreation facilities: obesogenic environments for children and families? Int J Environ Res Public Health. 2010;7(5):2208-21.

2. Naylor PJ, Olstad DL, Therrien S. An intervention to enhance the food environment in public recreation and sport settings: a natural experiment in British Columbia, Canada. Child Obes. 2015;11(4):364-74.

3. Chaumette P, Morency S, Royer A, Lemieux S, Tremblay A. Food environment in the sports, recreational and cultural facilities of Quebec City: a look at the situation. Can J Public Health. 2009;100(4):310-4.

4. Olstad DL, Raine KD, McCargar LJ. Adopting and implementing nutrition guidelines in recreational facilities: public and private sector roles. A multiple case study. BMC Public Health. 2012;12:376.

5. Driessen CE, Cameron AJ, Thornton LE, Lai SK, Barnett LM. Effect of changes to the school food environment on eating behaviours and/or body weight in children: a systematic review. Obes Rev. 2014;15(12):968-82.

6. Engler-Stringer R, Le H, Gerrard A, Muhajarine N. The community and consumer food environment and children's diet: a systematic review. BMC Public Health. 2014;14:522

7. Fine $L$, Philogene GS, Gramling R, Coups EJ, Sinha S. Prevalence of multiple chronic disease risk factors. 2001 National Health Interview Survey. Am J Prev Med. 2004;27(2 Suppl):18-24.

8. Pronk NP, Anderson LH, Crain AL, Martinson BC, O'Connor PJ, Sherwood NE, et al. Meeting recommendations for multiple healthy lifestyle factors. Prevalence, clustering, and predictors among adolescent, adult, and senior health plan members. Am J Prev Med. 2004;27(2 Suppl):25-33.

9. Nelson TF, Stovitz SD, Thomas M, Lavoi NM, Bauer KW, Neumark-Sztainer D. Do youth sports prevent pediatric obesity? A systematic review and commentary. Curr Sports Med Rep. 2011;10(6):360-70.

10. O'Connor SG, Koprowski C, Dzubur E, Leventhal AM, Huh J, Dunton GF. Differences in Mothers' and Children's dietary intake during physical and sedentary activities: an ecological momentary assessment study. J Acad Nutr Diet. 2017;117(8):1265-71.

11. McLeroy KR, Bibeau D, Steckler A, Glanz K. An ecological perspective on health promotion programs. Health Educ Q. 1988;15(4):351-77.

12. Hawkes $C$, Jewell J, Allen K. A food policy package for healthy diets and the prevention of obesity and diet-related non-communicable diseases: the NOURISHING framework. Obes Rev. 2013;14(Suppl 2):159-68.

13. British Columbia Ministry of Health. Healthier choices in vending machines in BC public buildings 2014 [Available from: https://www2.gov.bc.ca/assets/ gov/health/managing-your-health/healthy-eating/vending-policy-2014.pdf.

14. Alberta Health and Wellness. Alberta Nutrition Guidelines for Children and Youth: A childcare, school and recreation/community centre resource manual 2012 Available from: https://open.alberta.ca/publications/5906406.

15. Government of Nova Scotia. Healthy eating in recreation and sports settings 2015 Available from: https://thrive.novascotia.ca/node/83.

16. Mclsaac JL, Jarvis S, Olstad DL, Naylor PJ, Rehman L, Kirk SFL. Voluntary nutrition guidelines to support healthy eating in recreation and sports settings are ineffective: findings from a prospective study. AIMS Public Health. 2018;5(4):411-20.

17. Olstad DL, Downs SM, Raine KD, Berry TR, McCargar LJ. Improving children's nutrition environments: a survey of adoption and implementation of nutrition guidelines in recreational facilities. BMC Public Health. 2011;11:423.

18. Olstad DL, Raine KD, McCargar LJ. Adopting and implementing nutrition guidelines in recreational facilities: tensions between public health and corporate profitability. Public Health Nutr. 2013;16(5):815-23.

19. Mclsaac J, Jarvis SL, Spencer R, Kirk SFL. "A tough sell": findings from a qualitative analysis on the provision of healthy foods in recreation and sport settings. Health promotion and chronic disease prevention in Canada, vol. 38; 2018. p. 1

20. World Health Organization. The Bangkok charter for health promotion in a globalized world 2005 Available from: http://www.who.int/ healthpromotion/conferences/6gchp/bangkok_charter/en/.

21. Anderson J. The study of public policy. Public policymaking: an introduction. Boston: Houghton Mifflin Company; 2003. p. 2.

22. Sallis JF, Glanz K. Physical activity and food environments: solutions to the obesity epidemic. Milbank Q. 2009;87(1):123-54.
23. Olstad DL, Poirier K, Naylor PJ, Shearer C, Kirk SF. Policy outcomes of applying different nutrient profiling systems in recreational sports settings: the case for national harmonization in Canada. Public Health Nutr. 2015:18(12):2251-62.

24. Naylor PJ, Wekken SV, Trill D, Kirbyson A. Facilitating healthier food environments in public recreation facilities: results of a pilot project in British Columbia, Canada. J Park Recreat Adm. 2010;28(4):37-58.

25. Orlandi M, Landers C, Weston R, Haley N. Diffusion of health promotion innovations. In: Glanz K, Lewis F, Rimer B, editors. Health behavior and health education: theory, research and practice. San Francisco: Jossey-Bass Publishers; 1990. p. 288-313.

26. Robinson K, Elliott SJ, Driedger SM, Eyles J, O'Loughlin J, Riley B, et al Using linking systems to build capacity and enhance dissemination in heart health promotion: a Canadian multiple-case study. Health Educ Res. 2005:20(5):499-513.

27. Graham ID, Logan J, Harrison MB, Straus SE, Tetroe J, Caswell W, et al. Lost in knowledge translation: time for a map? J Contin Educ Heal Prof. 2006;26(1):13-24.

28. Durlak JA, DuPre EP. Implementation matters: a review of research on the influence of implementation on program outcomes and the factors affecting implementation. Am J Community Psychol. 2008;41(3-4):327-50.

29. Olstad DL, Goonewardene LA, McCargar LJ, Raine KD. Choosing healthier foods in recreational sports settings: a mixed methods investigation of the impact of nudging and an economic incentive. Int J Behav Nutr Phys Act. 2014;11(1):6.

30. Olstad DL, Goonewardene LA, McCargar $\sqcup$, Raine KD. If we offer it, will children buy it? Sales of healthy foods mirrored their availability in a community sport, commercial setting in Alberta, Canada. Child Obes. 2015;11(2):156-64.

31. Olstad DL, Vermeer J, McCargar LJ, Prowse RJ, Raine KD. Using traffic light labels to improve food selection in recreation and sport facility eating environments. Appetite. 2015;91:329-35.

32. British Columbia Healthy Living Alliance. Brand Name Food List [Available from: https://bnfl.healthlinkbc.ca/.

33. Partington SN, Menzies TJ, Colburn TA, Saelens BE, Glanz K. Reduced-item food audits based on the nutrition environment measures surveys. Am J Prev Med. 2015:49(4):e23-33.

34. Saelens BE, Glanz K, Sallis JF, Frank LD. Nutrition environment measures study in restaurants (NEMS-R): development and evaluation. Am J Prev Med. 2007;32(4):273-81

35. Hawkes C, Smith TG, Jewell J, Wardle J, Hammond RA, Friel S, et al. Smart food policies for obesity prevention. Lancet. 2015;385(9985):2410-21.

36. Gorski MT, Cohen JF, Hoffman JA, Rosenfeld L, Chaffee R, Smith L, et al Impact of nutrition standards on competitive food quality in Massachusetts middle and high schools. Am J Public Health. 2016; 106(6):1101-8.

37. Chriqui JF, Turner L, Taber DR, Chaloupka FJ. Association between district and state policies and US public elementary school competitive food and beverage environments. JAMA Pediatr. 2013;167(8):714-22.

38. Centers for Disease Control and Prevention. Results from the school health policies and practices study 20162016 Available from: https://www.cdc.gov/ healthyyouth/data/shpps/results.htm.

39. Woods J, Bressan A, Langelaan C, Mallon A, Palermo C. Australian school canteens: menu guideline adherence or avoidance? Health Promot J Austr. 2014;25(2):110-5.

40. Wolfenden L, Kingsland M, Rowland BC, Dodds P, Gillham K, Yoong SL, et al Improving availability, promotion and purchase of fruit and vegetable and non sugar-sweetened drink products at community sporting clubs: a randomised trial. Int J Behav Nutr Phys Act. 2015;12:35.

41. Brownson RC, Fielding JE, Green LW. Building capacity for evidence-based public health: reconciling the pulls of practice and the push of research Annu Rev Public Health. 2018:39:27-53.

42. Wolfenden L, Nathan N, Janssen LM, Wiggers J, Reilly K, Delaney T, et al. Multi-strategic intervention to enhance implementation of healthy canteen policy: a randomised controlled trial. Implement Sci. 2017;12(1):6.

43. Cobiac $L$, Veerman $L$, Vos $T$. The role of cost-effectiveness analysis in developing nutrition policy. Annu Rev Nutr. 2013;33:373-93.

44. Fielding JE. Health education 2.0: the next generation of health education practice. Health Educ Behav. 2013;40(5):513-9.

45. Moodie M, Sheppard L, Sacks G, Keating C, Flego A. Cost-effectiveness of fiscal policies to prevent obesity. Curr Obes Rep. 2013;2:211-24. 
46. Moodie R, Stuckler D, Monteiro C, Sheron N, Neal B, Thamarangsi T, et al. Profits and pandemics: prevention of harmful effects of tobacco, alcohol, and ultra-processed food and drink industries. Lancet. 2013;381 (9867):670-9.

47. Silberfarb LO, Savre S, Geber G. An approach to assessing multicity implementation of healthful food access policy, systems, and environmental changes. Prev Chronic Dis. 2014;11:E64.

48. Graff SK, Kappagoda M, Wooten HM, McGowan AK, Ashe M. Policies for healthier communities: historical, legal, and practical elements of the obesity prevention movement. Annu Rev Public Health. 2012;33:307-24.

49. Disantis Kl, Grier SA, Odoms-Young A, Baskin ML, Carter-Edwards L, Young DR, et al. What "Price" means when buying food: insights from a multisite qualitative study with black Americans. Am J Public Health. 2013;103(3):516-22.

50. Haynes-Maslow L, Parsons SE, Wheeler SB, Leone LA. A qualitative study of perceived barriers to fruit and vegetable consumption among low-income populations, North Carolina, 2011. Prev Chronic Dis. 2013;10:E34.

51. Slater J, Sevenhuysen G, Edginton B, O'Neil J. Trying to make it all come together': structuration and employed mothers' experience of family food provisioning in Canada. Health Promot Int. 2012;27(3):405-15.

52. Rafferty A, Gray VB, Nguyen J, Nguyen-Rodriguez S, Barrack M, Lin S. Parents report competing priorities influence snack choice in youth sports. J Nutr Educ Behav. 2018.

53. Simmons A, Reynolds RC, Swinburn B. Defining community capacity building: is it possible. Prev Med. 2011;52(3-4):193-9.

54. Leeman J, Calancie L, Kegler MC, Escoffery CT, Herrmann AK, Thatcher E, et al. Developing theory to guide building Practitioners' capacity to implement evidence-based interventions. Health Educ Behav. 2017:44(1):59-69

55. Irby MB, Drury-Brown M, Skelton JA. The food environment of youth baseball. Child Obes. 2014;10(3):260-5

56. Kelly B, Baur LA, Bauman AE, King L, Chapman K, Smith BJ. Examining opportunities for promotion of healthy eating at children's sports clubs. Aust N Z J Public Health. 2010;34(6):583-8.

57. Kelly B, Chapman K, King L, Hardy L, Farrell L. Double standards for community sports: promoting active lifestyles but unhealthy diets. Health Promot J Austr. 2008;19(3):226-8.

58. Boelsen-Robinson T, Chung A, Khalil M, Wong E, Kurzeme A, Peeters A. Examining the nutritional quality of food and beverage consumed at Melbourne aquatic and recreation centres. Aust N Z J Public Health. 2017:41(2):184-6.

59. Piggin J, Tlili H, Louzada BH. How does health policy affect practice at a sport mega event? A study of policy, food and drink at euro 2016. Int J Sport Policy Politics. 2017;9(4):739-51.

60. McFadyen T, Chai LK, Wyse R, Kingsland M, Yoong SL, Clinton-McHarg T, et al. Strategies to improve the implementation of policies, practices or programmes in sporting organisations targeting poor diet, physical inactivity, obesity, risky alcohol use or tobacco use: a systematic review. BMJ Open. 2018;8(9):e019151.

61. Movassagh EZ, Baxter-Jones ADG, Kontulainen S, Whiting SJ, Vatanparast H. Tracking dietary patterns over 20 years from childhood through adolescence into Young adulthood: the Saskatchewan pediatric bone mineral accrual study. Nutrients. 2017;9(9):990.

62. Mikkila V, Rasanen L, Raitakari OT, Pietinen P, Viikari J. Consistent dietary patterns identified from childhood to adulthood: the cardiovascular risk in Young Finns study. Br J Nutr. 2005;93(6):923-31.

63. Vander Wekken S, Sorensen S, Meldrum J, Naylor PJ. Exploring industry perspectives on implementation of a provincial policy for food and beverage sales in publicly funded recreation facilities. Health Policy. 2012;104(3):279-87.

64. Roncarolo F, Lanati N, Philpott M, Drygas W, Ruszkowska J, Ireland R, et al. Process evaluation of European 'Healthy Stadia' program. Health Promot Int. 2015:30(4):881-90.

65. Meganck J, Seghers J, Scheerder J. Exploring strategies to improve the health promotion orientation of Flemish sports clubs. Health Promot Int. 2017;32(4):681-90

66. Statistics Canada. National Household Survey 2011 [Available from: http:// www12.statcan.gc.ca/nhs-enm/2011/dp-pd/prof/index.cfm?Lang=E.

67. Statistics Canada. From urban areas to population centres 2011 [Available from: http://www.statcan.gc.ca/eng/subjects/standard/sgc/notice/sgc-06.

\section{Publisher's Note}

Springer Nature remains neutral with regard to jurisdictional claims in published maps and institutional affiliations.

Ready to submit your research? Choose BMC and benefit from:

- fast, convenient online submission

- thorough peer review by experienced researchers in your field

- rapid publication on acceptance

- support for research data, including large and complex data types

- gold Open Access which fosters wider collaboration and increased citations

- maximum visibility for your research: over $100 \mathrm{M}$ website views per year

At BMC, research is always in progress.

Learn more biomedcentral.com/submissions 